\title{
Look. Up in the Sky... It's a Bird, It's a Plane, It's a Bear!
}

\author{
Brian Hayden \\ University of British Columbia \\ bhayden@sfu.ca
}

While hunter-gatherers in general form a baseline out of which all astronomical systems developed, it is probably really only with the emergence of complex hunter-gatherers that relatively complex astronomical systems emerge (Hayden and Villeneuve 2011). We can wonder how far back in time the identification of star configurations existed as constellations, and even how far back in time recognition of specific constellations may have occurred, especially those that lay along the ecliptic or in relation to special areas of the sky such as the poles, or as unusually distinctive configurations such as the Pleiades, Taurus or Orion's Belt. In trying to wrestle with such questions, there are two types of data that are of most use: firstly, ethnographic observations from hunting and gathering societies, and secondly, prehistoric images that may be related to star clusters or constellations. Unfortunately, because detailed astronomical knowledge was some of the most closely guarded esoteric knowledge of secret societies - sometimes with death penalties for any members who divulged such secrets - the information recorded by ethnographers often only consisted of superficial common knowledge of astronomical systems in cultures of complex hunter-gatherers. However, there are at least a few exceptions, such as the observations that were recorded for the Chumash by Hudson and Underhay (1978), which should be used for their full worth.

While Villeneuve and I (Hayden and Villeneuve 2011) have conducted a preliminary assessment of selected hunter-gatherer constellation concepts, our initial conclusions were far from definitive, and a much more systematic comparative study needs to be undertaken both for simple hunter-gatherers, or "foragers", and for more complex hunter-gatherers. One of the more interesting results from our initial survey was the strong tendency, especially among simple hunter-gatherers, to view individual stars as animals, people or objects, while distinctive configurations of stars tended to be viewed as groups of animals, people or objects (see also Aujoulat 2004). Such simple sky maps could conceivably go back to Middle Paleolithic times, or perhaps even earlier. Constel- 
lations in the modern sense of star configurations representing individual animals or people, where they existed, usually occurred as minor aspects of sky maps among simple foragers. Thus, the earliest sky maps may have simply viewed individual stars as individual beings, much as the Romans viewed dead souls as ascending to the sky, where they became individual stars in the Milky Way. If this was so, it becomes interesting to think about when contemporary types of constellations emerged.

There are a number of indications that star configurations representing individuals probably developed at least in the Upper Paleolithic, in the context of complex huntergatherer societies which were likely characterised by the development of a more complex or esoteric ideology and mythology associated with the emergence of secret societies or similar ritual organisations. Among others, Jeguès-Wolkiewiez (2000; 2005; 2007; 2008), Rappenglück (1999; 2000) and Aujoulat (2004) have argued for various representations of constellations in Upper Paleolithic cave art, the most notable being the cluster of dots resembling the Pleiades immediately to the right of a Taurus-like bull's head in the entry chamber of Lascaux Cave in France.

Ethnographically documented constellations and more complex astronomical observations seem to support the scenario of star configurations conceived as individual images as being common among complex hunter-gatherers. However, this again is a preliminary conclusion which merits more detailed and systematic study. A relatively new approach which can be used to estimate the historical depth of constellations (in the contemporary sense) is similar to the methods used by historical linguists to reconstruct paleo-languages. As in genetics, in essence, this assumes that the most widespread contemporary motifs were derived from similar ancestral forms. The method consists of finding the most common linguistic, genetic or constellation forms in a given geographical area, and reconstructing past linguistic or other systems on the basis of the most common elements that can be identified. In essence, this is the method that we used when we proposed that Ursa Major and Polaris probably originated at least in the Upper Paleolithic, if not earlier. In this case, the most common elements were that the individual stars in Ursa Major represented individual hunters chasing a bear (Polaris). Very similar concepts for this constellation were present in a surprising variety of cultures in North America and Eurasia, including the identification of Ursa Major and Minor with bears by the Greeks. Similar studies are certainly warranted for other constellations including Taurus, the Pleiades, Orion, Pegasus and Auriga.

Recent research by d'Huy $(2012 ; 2013 ; 2016)$ has pioneered a systematic way of measuring the genetic (historical) proximity of myths associated with constellations. His techniques have produced graphs displaying similarity relationships and distances (Figure 1). When combined with geographic distributions and known colonisation timeframes, these graphed relationships can be used to roughly estimate time depths involved. His study has applied this methodology to the hunting myths associated with the constellation of Ursa Major using genetics-based mathematical and graphic modeling (d'Huy 2012), with surprising results. The relationships between these myths in a variety of cultures leads to the conclusion that the basic myth is older than 15,000 years and diffused into the Americas from Central Asia. This kind of approach provides very fertile 
ground for future astronomical research, and has been extended to the Pleiades and the Milky Way (d'Huy 2017). Similar studies are certainly warranted for other constellations including Taurus, Orion, Pegasus, Cassiopeia and Auriga.

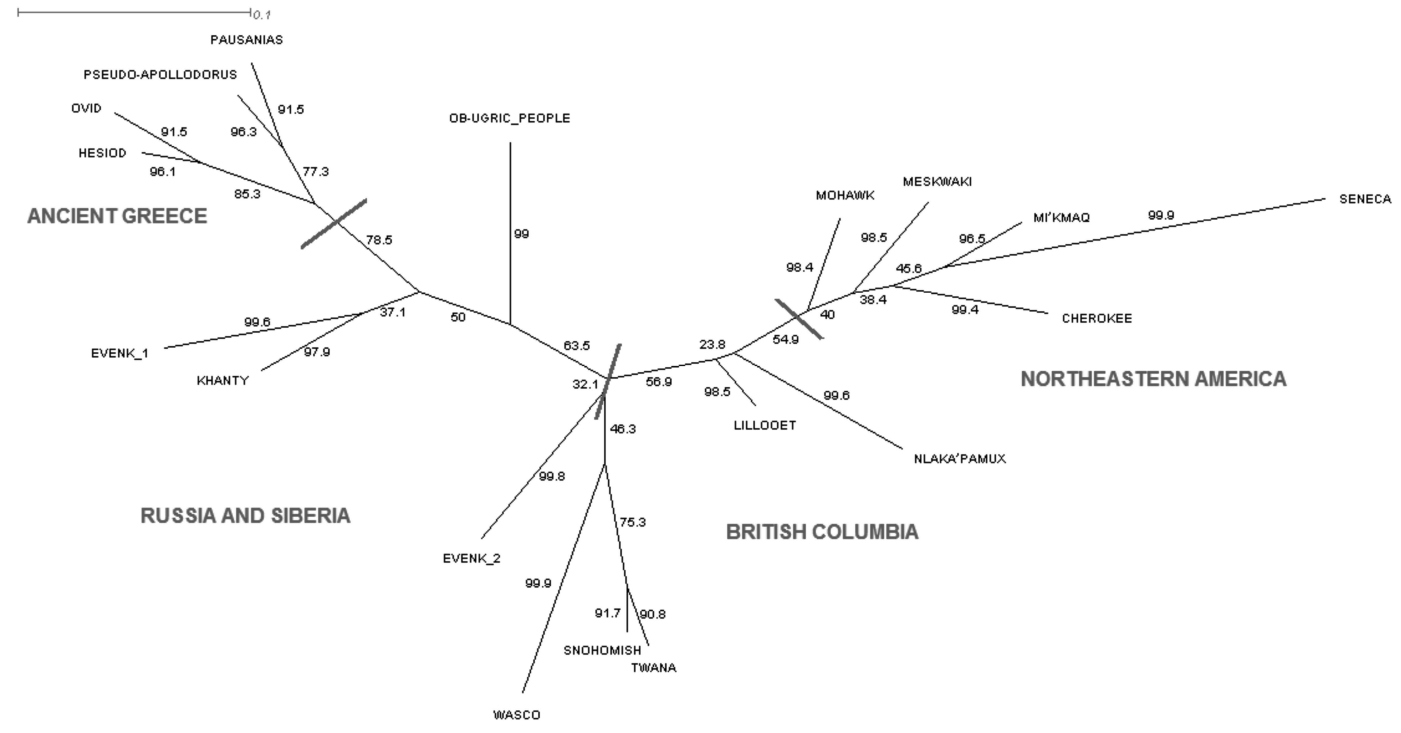

FIGURE 1. A tree diagram based on the calculated similarities of the "Cosmic Hunt" myth associated with Ursa Major (d'Huy 2012, 99).

Using ethnographic sources, I would like to draw attention to one particular type of cave art image from the Upper Paleolithic that has long remained enigmatic for prehistorians but which has good potential for understanding constellations of the period. These prehistoric images are referred to as "tectiforms", essentially pentagonal shapes that have been viewed most frequently as tent structures. It is important to recognise that the standard form of mobile hunter-gatherer tents is conical or a dome-shaped structure that in both instances have circular ground plans. Tectiforms appear to depict a very different type of structure, square in plan with vertical walls and a peaked roof (Figure 2).

Thus, assuming that tectiforms do represent some type of structure, they would appear to be special types of structures. Hayden and Villeneuve (2011) have suggested that the most likely candidate would be what is called by Algonkian Indians in North America a "shaking tent", specially constructed with vertical walls for shamanic trances. Intriguingly, when the Algonkian shaman enters these shaking tents, he throws powders out of the top and he imagines connections with the upper or spirit world (Figure 3), allowing him to travel to the sky and enter the Sky World, typically through the portal represented by Polaris (Hoffman 1891, 252). Similar practices may exist in Siberia and could certainly have been part of the shamanic or secret society repertory of Upper Paleolithic groups in Europe. Several of the tectiform images depict materials spewing out of the tops of the structures (see Figure 2). 

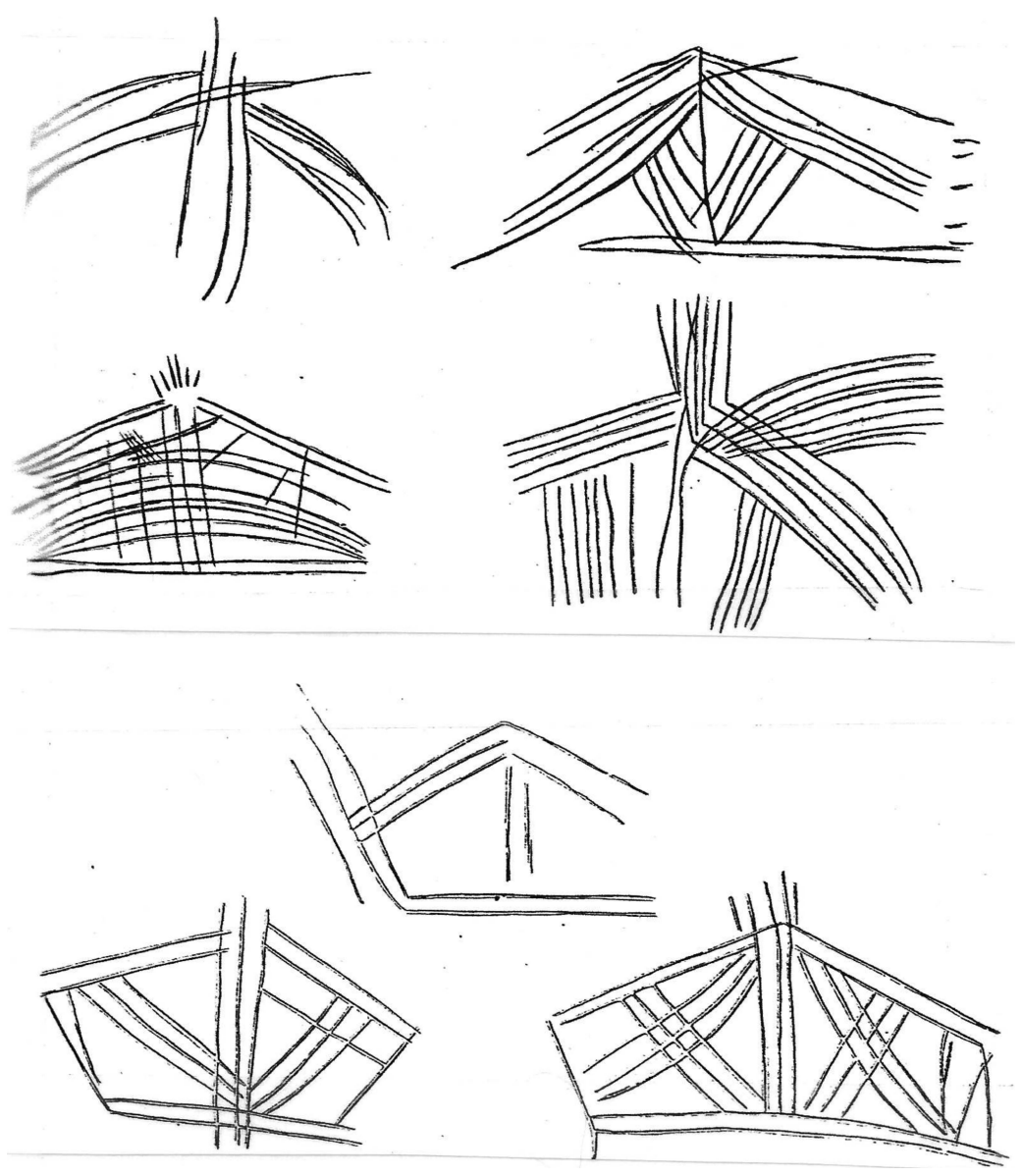

FIGURE 2. Examples of tectiforms from the Upper Paleolithic cave of Font-de-Gaume in France (Capitan et al. 1916: 228-229, 231). Note particularly the images showing something coming out of the peak of the structure.

Coincidentally, there is a constellation which bears a strong resemblance to the tectiforms in Upper Paleolithic caves: Auriga. The potential significance of Auriga is enhanced when it is realised that not only is it within the general orbit of Polaris, but that two of its star alignments point either directly to Polaris or to its general vicinity (Figure 4). The Milky Way also passes through part of Auriga. This constellation, therefore, could have been used in the past, much as the stars of Ursa Major are used today, as locators for Polaris. An association between the shape of shamanic shaking tents and the shape of Auriga, as well as between shamanic travel from the shaking tents to Polaris and the alignment of Auriga stars with Polaris, seems far from a haphazard coincidence. Thus, I think that a good argument can be made for the importance of the Auriga constellation in the sky maps of the Upper Paleolithic and one of the first examples of a complex star configuration representing an entire object or being, plausibly forming part of secret society esoteric ideology and mythology. 

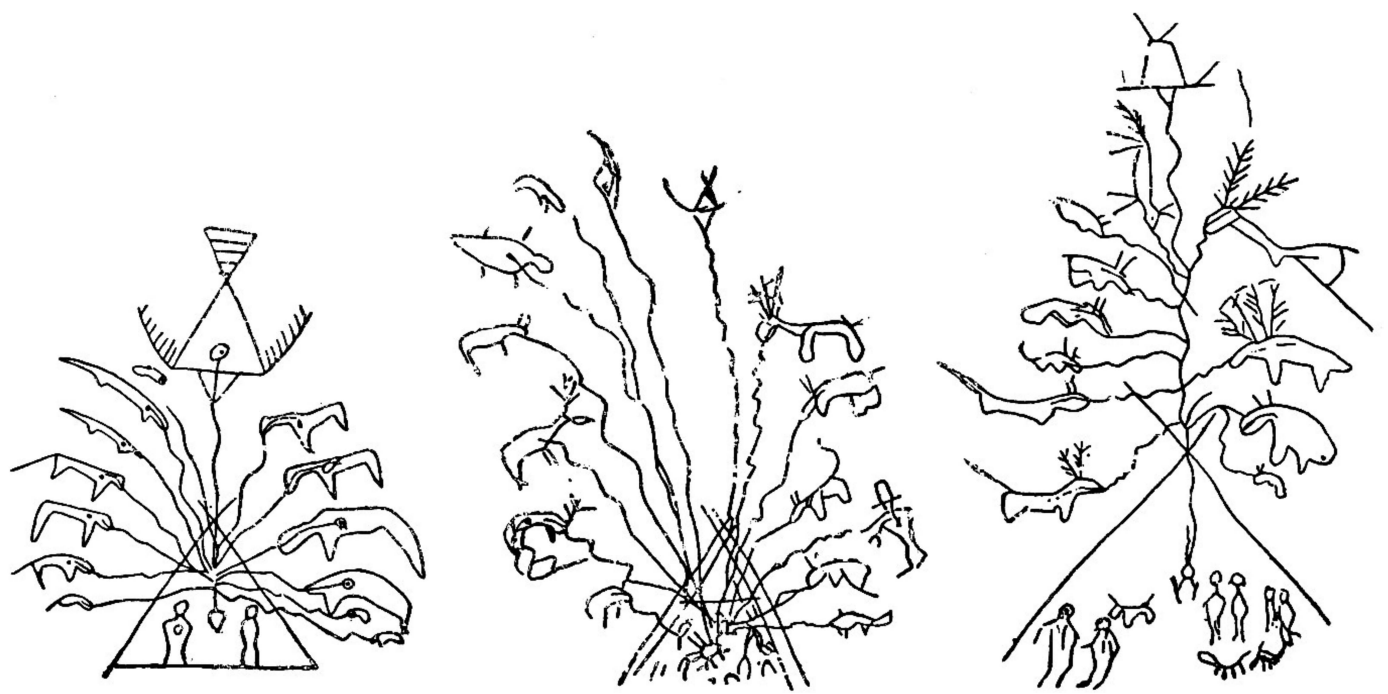

FIGURE 3. Tents used by high ranking Ojibway members of the Midewiwin society (Hoffman 1891, 252), indicating spiritual connections emanating from the top of the tent to the cosmos. Although these depictions are of the residential tents used by these men, similar concepts must have also been part of their use of shaking tents (with vertical walls), which were portals to the astral world, especially via Polaris.

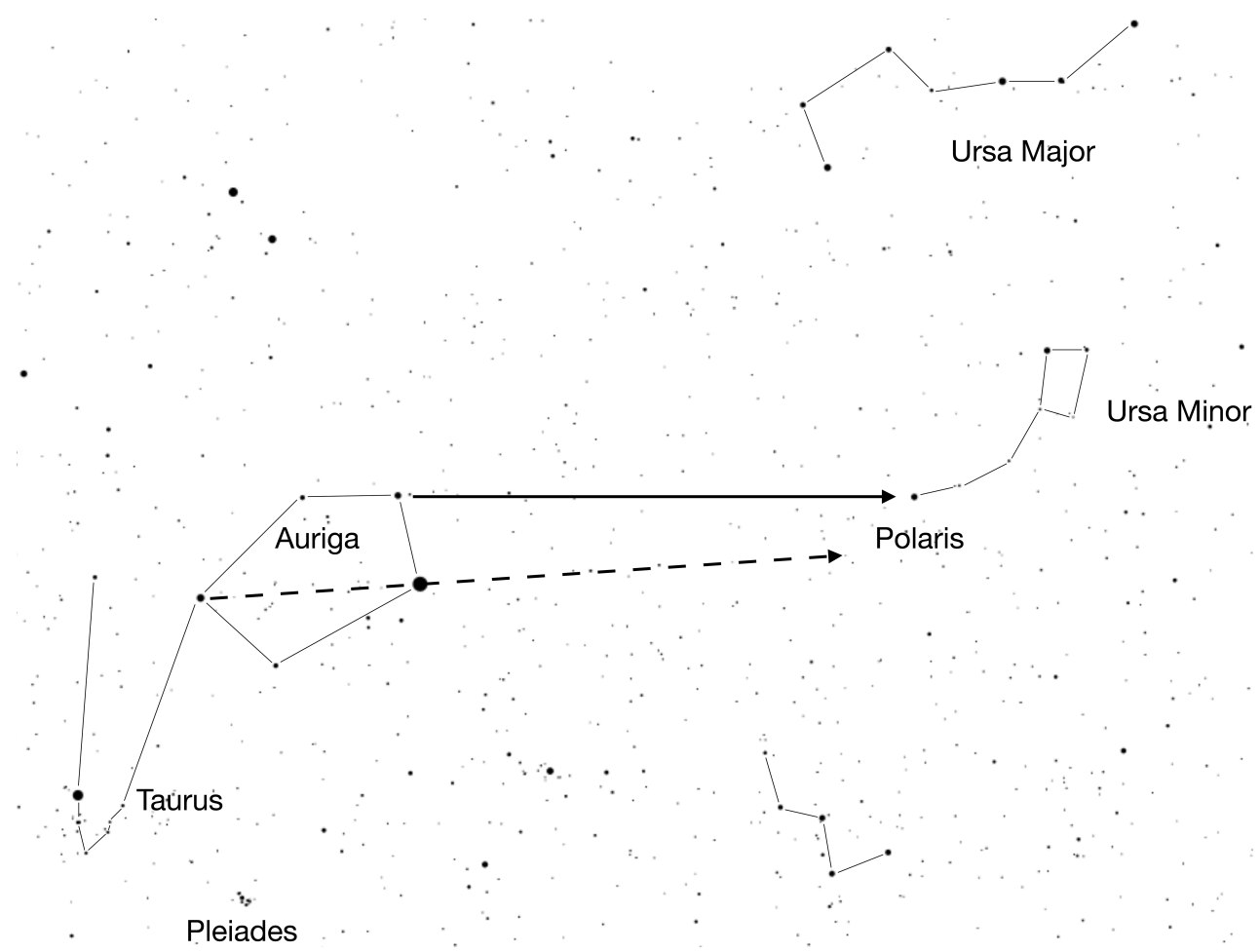

FIGURE 4. The relation of Auriga to Polaris, indicating the major star alignments that point to or converge at Polaris. 
In conclusion, I would like to stress the great potential that the stars hold for understanding prehistoric societies. While the Sun, the Moon and the planets have been favoured as the lowest hanging astral bodies to pick in this regard, the stars and constellations promise to be fruitful in other important ways.

\section{Acknowledgements}

I want to express my gratitude to Fabio Silva and others at the Journal of Skyscape Archaeology who have invited this submission and helped to illustrate it. My thanks also go to Julien d'Huy who generously shared his research with me and provided an illustration for this submission.

\section{References}

Aujoulat, N., 2004. Lascaux, Le Geste, L'Espace et le Temps. Paris: Seuil.

Capitan, L., H. Breuil and D. Peyrony, 1916. La Caverne de Font-de-Gaume aux Eyzies (Dordogne). Monaco: A. Chène.

D’Huy, J., 2012. "Un ours dans les étoiles: Recherche phylogénétique sur un mythe préhistorique". Bulletin, Préhistoire du Sud-Ouest 20: 91-106.

D'Huy, J., 2013. "A Cosmic Hunt in the Berber Sky: A Phylogenetic Reconstruction of a Palaeolithic Mythology". Les Cahiers de I'Association des Amis de l'Art Rupestre Saharien 16: 93-106.

D’Huy, J., 2016. "The Evolution of Myths". Scientific American 315 (December): 63-69. https://doi.org/10.1038/ scientificamerican1216-62

D’Huy, J., 2017. "Lascaux, les Pléiades, et la Voie Lactée”. Mythologie Française 267: 19-22.

Hayden, B. and S. Villeneuve, 2011. "Astronomy in the Upper Paleolithic?" Cambridge Archaeological Journal 21 (3): 331-355. https://doi.org/10.1017/S0959774311000400

Hoffman, W. J., 1891. "The Mide'wiwin or 'Grand Medicine Society' of the Ojibwa". In Seventh Annual Report of the Bureau of Ethnology to the Secretary of the Smithsonian Institution, 1885-1886, 149-300. Washington, DC: US Government Printing Office.

Hudson, T. and E. Underhay, 1978. Crystals in the Sky: An Intellectual Odyssey Involving Chumash Astronomy, Cosmology and Rock Art. Socorro, New Mexico: Ballena Press.

Jeguès-Wolkiewiez, C., 2000. "Lascaux, vision du ciel des Magdaléniens". In Proceedings XVIII Valcamonica Symposium: Arte rupestre e tribale: Conservazione e salvaguardia dei messaggi, edited by E. Anati, 29.

Capo di Ponte, Italy: Ediciones Del Centro.

Jeguès-Wolkiewiez, C., 2005. "Aux racines de l'astronomie ou l'ordre caché d'une oeuvre paléolithique". Antiquités Nationales 37: 43-52.

Jeguès-Wolkiewiez, C., 2007. “Cro-Magnon: premier astronome de l'humanité?" Science et Vie 1087: 93-101. Jeguès-Wolkiewiez, C., 2008. "Lascaux et les astres". Les Dossiers d'Archaeologie (Hors Série) 15: 22-29.

Rappenglück, M. A., 1999. "Palaeolithic Timekeepers Looking at the Golden Gate of the Ecliptic: The Lunar Cycle and the Pleiades in the Cave of La-Tête-du-Lion (Ardèche, France) - 21,000 BP". Earth, Moon and Planets 85-86: 391-404. https://doi.org/10.1023/A:1017069411495

Rappenglück, M. A., 2000. "Ice Age People Find their Ways by the Stars: A Rock Picture in the Cueva de El Castillo (Spain) May Represent the Circumpolar Constellation of the Northern Crown (CrB)". Migration and Diffusion: An International Journal 1 (2): 15-28.

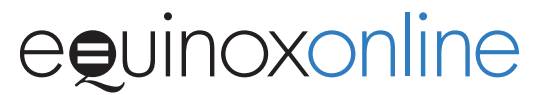

